\title{
Thymol ameliorates ammonium toxicity via repressing polyamine oxidase-derived hydrogen peroxide and modulating ammonium transporters in rice root
}

Kai Guo ${ }^{1,2+}$, Guangchi An ${ }^{3+}$, Ning Wang ${ }^{4}$, Bingdong Pang ${ }^{5}$, Zhiqi Shi ${ }^{5}$, Hongwu Bai $^{5}$, Li Zhang ${ }^{6}$, Jian Chen ${ }^{5^{*}}$ (D) and Weimin $\mathrm{Xu}^{7}$

\begin{abstract}
Background: Ammonium is an indispensable nutrient for crop growth, but anoxic conditions or inappropriate fertilizer usage result in the increase in ammonium content in soil. Excessive ammonium causes phytotoxicity. Thymol is a kind of natural phenolic compound with anti-microbial properties. However, little is known about the role of thymol in modulating plant physiology. Here we find the novel role of thymol in protecting rice from ammonium toxicity.

Results: Thymol remarkably rescued rice seedlings growth from ammonium stress, which may resulted from the attenuation of reactive oxygen species (ROS) accumulation, oxidative injury, and cell death in both shoots and roots. Polyamine oxidase (PAO) metabolizes polyamines to produce ROS in plants in response to stress conditions. Thymol blocked ammonium-induced upregulation of a set of rice PAOs, which contributed to the decrease in ROS content. In rice seedlings upon ammonium stress, thymol downregulate the expression of ammonium transporters (AMT1;1 and AMT1;2); thymol upregulated the expression of calcineurin B-like interacting protein kinase 23 (CIPK23) and calcineurin B-like binding protein 1 (CBL1), two negative regulators of AMTs. This may help rice avoid ammonium overload in excessive ammonium environment. Correlation analysis indicated that PAOS, AMTs, and CBL1 were the targets of thymol in the detoxification of excessive ammonium.
\end{abstract}

Conclusion: Thymol facilitates rice tolerance against ammonium toxicity by decreasing PAO-derived ROS and modulating ammonium transporters. Such findings may be applicable in the modulation of nutrient acquisition during crop production.

Keywords: Ammonium toxicity, Polyamine oxidase, Reactive oxygen species, Rice, Thymol

\footnotetext{
* Correspondence: chenjian@jaas.ac.cn

${ }^{\dagger}$ Kai Guo and Guangchi An contributed equally to this work.

5 Institute of Food Safety and Nutrition, Jiangsu Academy of Agricultural

Sciences / Laboratory for Food Quality and Safety-State Key Laboratory

Cultivation Base of Ministry of Science and Technology, Nanjing 210014,

China

Full list of author information is available at the end of the article
}

(c) The Author(s). 2021 Open Access This article is licensed under a Creative Commons Attribution 4.0 International License, which permits use, sharing, adaptation, distribution and reproduction in any medium or format, as long as you give appropriate credit to the original author(s) and the source, provide a link to the Creative Commons licence, and indicate if changes were made. The images or other third party material in this article are included in the article's Creative Commons licence, unless indicated otherwise in a credit line to the material. If material is not included in the article's Creative Commons licence and your intended use is not permitted by statutory regulation or exceeds the permitted use, you will need to obtain permission directly from the copyright holder. To view a copy of this licence, visit http://creativecommons.org/licenses/by/4.0/. 


\section{Introduction}

Ammonium $\left(\mathrm{NH}_{4}{ }^{+}\right)$is one of the major sources for crops acquiring nitrogen. Applying artificial fertilizer is the main approach to provide nitrogen for crops due to the decrease in agricultural soil fertility and low nitrogen use efficiency of crops. However, the inappropriate fertilizer usage with imbalanced nutrients, e.g. the prolonged usage of ammonium as sole nitrogen source, results in the increase in soil ammonium level. In addition, soils at anoxic conditions (e.g. rice paddies) or low $\mathrm{pH}$ conditions are always rich in ammonium because of the high rate of ammonification. In these soils, applying other form of nitrogen (e.g. nitrate) can also increase ammonium level (Chen et al. 2013; Esteban et al. 2016). Ammonium sensitivity of crops has been drawing great attention worldwide. High level of ammonium induces crop physiological disorders, resulting in growth inhibition and yield losses (Esteban et al. 2016). Ammoniuminduced phytotoxicity has been linked to the disturbance of multiple physiological processes (Gerendás et al. 1997). One of them is oxidative injury that is resulted from reactive oxygen species (ROS) accumulation in plant cells (Xie et al. 2015). Therefore, rescuing plant from oxidative damage could be useful for modulating of ammonium tolerance. Ammonium stress represses the activities of anti-oxidative enzymes in plants, resulting in compromised ability to scavenge already over-generated ROS (Xie et al. 2015). However, how ammonium triggers ROS overgeneration in plants remains elusive. Polyamine oxidase (PAO)-mediated deamination metabolism of polyamines is an important biosynthetic pathway of hydrogen peroxide $\left(\mathrm{H}_{2} \mathrm{O}_{2}\right)$, one of typical $\mathrm{ROS}$, in plant development and stress responses (Alcázar and Tiburcio 2014). Total of seven PAO genes (PAO1-7) have been identified in rice. ROS produced from these PAOs play a role in the regulation of rice seed germination (Chen et al. 2016). Modifying nitrogen sources can affect plant endogenous polyamines level (Altman and Levin 1993), but little is known about whether ammonium triggers polyamines metabolism to produce ROS.

Another approach to protect crop from ammonium toxicity is to modulate ammonium uptake under high level of ammonium conditions. A set of membrane proteins named ammonium transporters (AMTs) facilitate the uptake and transport of ammonium in plants (Li et al. 2012). AMT genes show diverse expression patterns in different tissues, but many of them can be activated and induced by ammonium (Sonoda et al. 2003; Li et al. 2012). Plants have developed intrinsic strategies to regulate AMT to avoid ammonium overload. For instance, calcineurin B-like interacting protein kinase 23 (CIPK23) and calcineurin B-like binding protein 1 (CBL1) are two negative regulators for ammonium transport. CIPK23 interacts with CBL1 to repress the activity of AMT1;1 and AMT1;2, leading to the inhibition of ammonium transport (Straub et al. 2017). Therefore, CIPK23CBL1-AMT module is an important regulatory node for the modulation of ammonium uptake.

Thymol is a kind of natural compound found in Thymus species (Basch et al. 2004). Thymol has been used as food preservative and potential medicine based on its anti-micorbial and anti-oxidative properties (Marchese et al. 2016; Nagoor Meeran et al. 2016). Thymol has been considered as a potential candidate of safety agrochemicals (USEPA/IRIS 2014), but little is known about its possible function on the regulation of crop physiology. Our previous study indicated that thymol was able to regulate rice nitric oxide signaling to decrease ROS accumulation in order to combat abiotic stress (Wang et al. 2017). This drove us to think about whether thymol could regulate plant tolerance against ammonium toxicity.

In this work, we investigated the role of thymol in ammonium detoxification in rice seedlings. Thymolameliorated ROS generation and oxidative injury were associated with the repression of PAOs transcription in rice seedlings under ammonium stress. Then we examined the effect of thymol on the expression of ammonium transporters and their regulators. Finally, we discussed the correlation among different parameters, the possible mechanism for thymol-conferred ammonium tolerance, and its significance.

\section{Materials and methods}

Plant culture, treatment, and chemicals

Rice (Oryza sativa) seeds (Nangeng 9108, obtained from Institute of Food Crops, Jiangsu Academy of Agricultural Sciences, China) were surface-sterilized with $1 \% \mathrm{NaClO}$ solution for 10 mins followed by washed with distilled water. Then the seeds were soaked with distilled water for $12 \mathrm{~h}$, and were placed on a floating plastic net for germination in darkness. Thirty identical seedlings with root length about 1.5 $\mathrm{cm}$ were selected to transfer into a container with $1 \mathrm{~L}$ modified Kimura B nutrient solution ( $\mathrm{Hu}$ et al. 2015). The rice seedlings were grown in a chamber with temperature at $28^{\circ} \mathrm{C}$, photosynthetic active radiation of $200 \mu \mathrm{mol} / \mathrm{m}^{2} / \mathrm{s}$, and photoperiod of $12 \mathrm{~h}$. $\mathrm{NH}_{4} \mathrm{Cl}$ and thymol at different concentrations were added to the nutrient solution up to $72 \mathrm{~h}$ according to different experimental designs.

Thymol at analytical purity (>99\%) were obtained from Sigma-Aldrich (St. Louis, MO). All the fluorescent 
probes used in this study were obtained from Beyotime Biotechnology Institute (Haimen, China). All the other chemicals at analytical grade were obtained from Sinopharm Chemical Reagent Co., Ltd. (Shanghai, China).

\section{Histochemical analysis}

Total ROS, $\mathrm{O}_{2}{ }^{--}$(superoxide radical anion), $\mathrm{H}_{2} \mathrm{O}_{2}$, and cell death in root tips were detected in situ using specific fluorescent probe DCFH-DA $\left(2^{\prime}, 7^{\prime}\right.$-dichlorofluorescein diacetate), DHE (dihydroethidium), HPF [3'-(p-hydroxyphenyl) fluorescein], and PI (propidium iodide), respectively, followed by capturing fluorescent images and calculating fluorescent density based on our previously published methods (Ye et al. 2017). The fluorescence was observed and captured with a fluorescence microscope (ECLIPSE, TE2000-S, Nikon, Melville, NY, USA) with setting of excitation $480-490 \mathrm{~nm} /$ emission $515-525$ $\mathrm{nm}$ for green fluorescence (DCFH-DA and HPF) and excitation $530-540 \mathrm{~nm} / \mathrm{emission} 610-620 \mathrm{~nm}$ for red fluorescence (DHE and PI). Histochemical staining of leaves was performed based on our previous study (Ye et al. 2016). $\mathrm{O}_{2}{ }^{--}, \mathrm{H}_{2} \mathrm{O}_{2}$, lipid peroxidation, loss of membrane integrity, and cell death in leaves were evaluated in vivo by staining with NBT (nitro-blue tetrazolium), DAB (3, 3-diaminobenzidine), Schiff's reagent, Evans blue, and Trypan blue, respectively, followed by removing chlorophyll background with ethanol in boiling water. The leaves after staining were observed and photographed by using a stereoscopic microscope (SteREO Discovery.V8, ZEISS, Oberkochen, Germany).

\section{Measurement of TBARS content}

TBARS (thiobarbituric acid reactive substances) content in plant tissues was measured by using a commercial TBARS kit (A003; Nanjing Jiancheng Bioengineering Institute, Nanjing, China), based on the spectrophotometrical measurement of the reaction between TBARS and TBA (1,3-diethyl-2-thiobarbituric acid) in the presence of TCA (trichloroacetic acid) according to manufacturer's instructions. The absorbance at $532 \mathrm{~nm}$ of the reaction product was recorded for the calculation of the final TBARS concentration based on manufacturer's instructions (Yang et al. 2012).

\section{Gene expression analysis}

The relative expression level of rice genes was obtained with qRT-PCR (real-time quantitative reverse transcription polymerase chain reaction). Total RNA were extracted form shoots and roots, respectively, by using Trizol (Invitrogen). Then cDNA was obtained from reverse transcription by using PrimeScript first Strand cDNA Synthesis Kit (TaKaRa Bio Inc., Dalian, China). The real-time quantitative PCR was performed using Applied Biosystems 7500 Fast RealTime PCR System (Applied Biosystems, Waltham, MA, USA) with SYBR Premix Ex Taq ${ }^{\text {Tm }}$ (TaKaRa Bio Inc., Dalian, China). Data were collected and analyzed by using ABI 7500 software (v. 2.0.6, Applied Biosystems) based on $2^{-\triangle \Delta C T}$ threshold cycle method (Livak and Schmittgen 2001). The relative abundance of house-keeping gene Actin was used as the internal standard to normalize the expression data. The detailed procedure can be found in our previous study (Ye et al. 2016). The final expression data was clustered and presented as heatmap using Cluster 3.0 combined with Java Treeview (Shi et al. 2014). The sequences of primers for gene amplification were listed in Table S1.

\section{Statistical analysis}

Each result was presented as mean \pm SD (standard deviation) of at least three replicates. Least significant difference test (LSD) was performed on data following ANOVA (one-way analysis of variance) tests to test for significant $(P<0.05)$ differences among treatments. Pearson correlation analysis among different parameters was performed using package "corrplot" in R (Wei and Simko 2017).

\section{Results and discussion}

Thymol attenuated growth stunt and oxidative injury in rice seedlings upon ammonium stress

We first evaluated the toxic effect of excessive $\mathrm{NH}_{4} \mathrm{Cl}$ on rice seedlings by measuring the length of root and shoot, respectively, upon the exposure of $\mathrm{NH}_{4} \mathrm{Cl}$ at different concentrations (10-80 mM). Treatment with $\mathrm{NH}_{4} \mathrm{Cl}$ for $72 \mathrm{~h}$ led to remarkable decrease in both root length and shoot length in dose-dependent manners (Fig. 1A and B). Thymol was added to the treatment solution in the presence of $20 \mathrm{mM} \mathrm{NH} \mathrm{NH}_{4} \mathrm{Cl}$ that caused moderate toxicity to seedling growth. Treatment with thymol $(5-40 \mu \mathrm{M})+$ $\mathrm{NH}_{4} \mathrm{Cl}$ led to significant increase in root length compared with $\mathrm{NH}_{4} \mathrm{Cl}$ treatment alone (Fig. S1A). Thymol at $20 \mu \mathrm{M}$ showed the best effect (Fig. S1A; Fig. 1C). Similar results were also found for shoot length (Fig. S1B; Fig. 1D). Therefore, thymol at $20 \mu \mathrm{M}$ was selected in the following experiments. In time-course experiments, thymol began to significantly rescue the growth of root and shoot at $6 \mathrm{~h}$, further giving rise to increased growth speed than that of $\mathrm{NH}_{4} \mathrm{Cl}$ treatment alone (Fig. $1 \mathrm{E}$ and $\mathrm{F}$ ). These results suggested that thymol was able to recover the growth of rice seedlings from $\mathrm{NH}_{4} \mathrm{Cl}$ stress.

Since ammonium-induced phytotoxicity is always associated with oxidative injury (Xie et al. 2015), we 

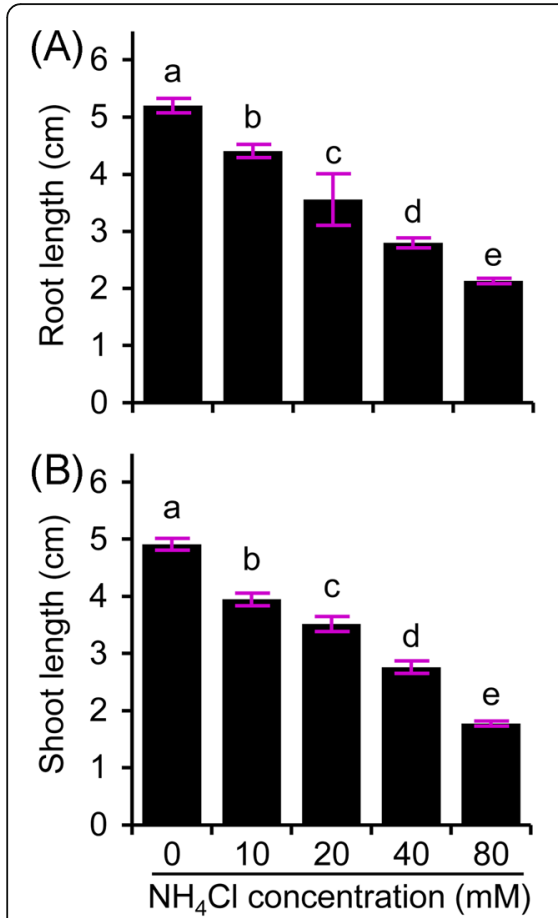
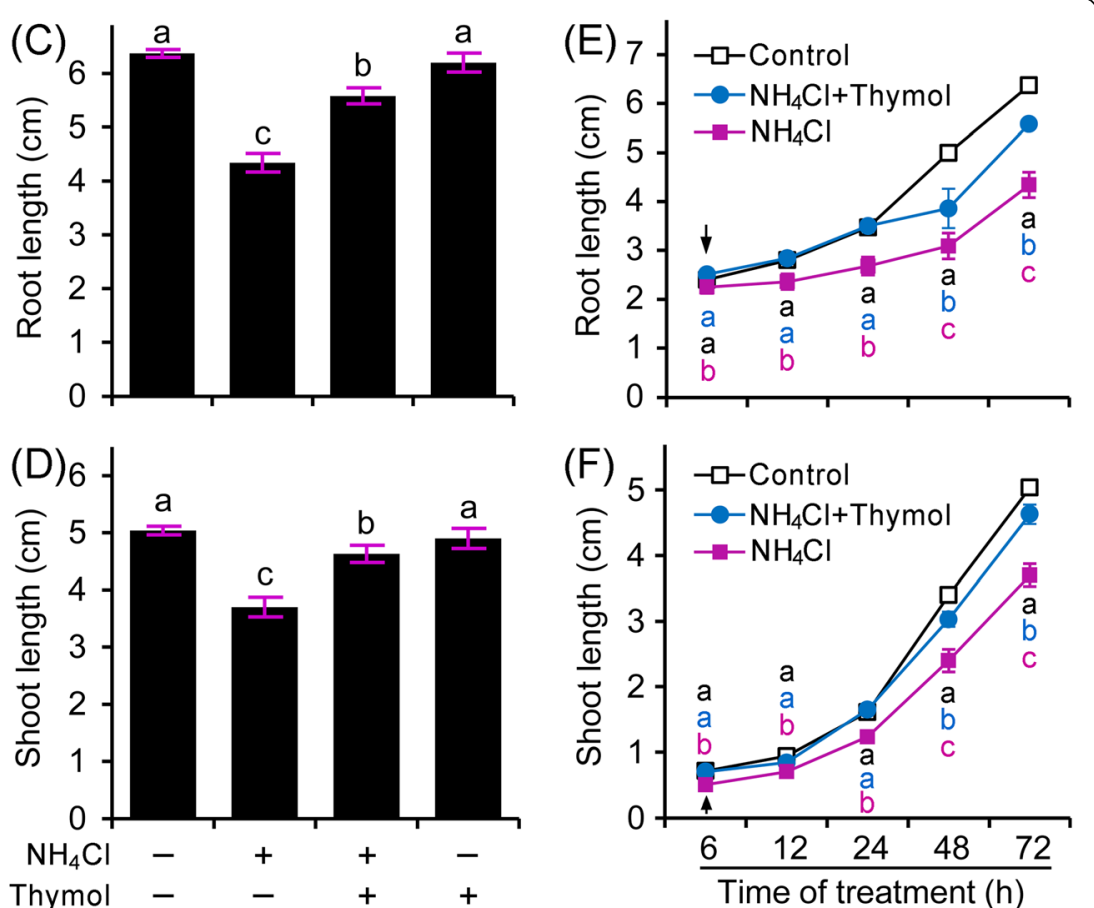

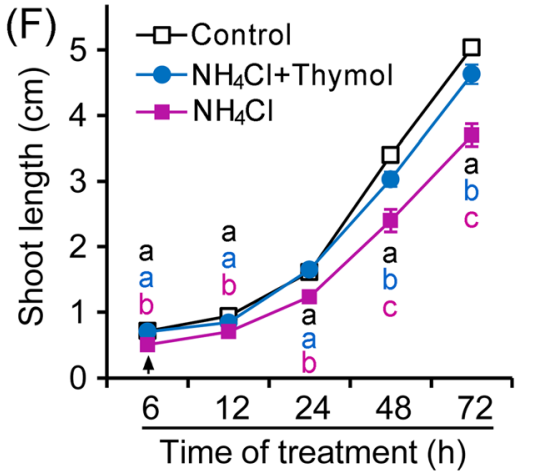

Fig. 1 Thymol attenuated ammonium-induced growth inhibition in rice seedlings. (A-B) Changes in the length of root and shoot after $\mathrm{NH}_{4} \mathrm{Cl}$ exposure for $72 \mathrm{~h}$. (C-D) Changes in the length of root and shoot after $\mathrm{NH}_{4} \mathrm{Cl}(20 \mathrm{mM})$ exposure with or without thymol (20 $\left.\mu \mathrm{M}\right)$ for $72 \mathrm{~h}$. (E-F) Time-dependent changes in root length and shoot length after $\mathrm{NH}_{4} \mathrm{Cl}(20 \mathrm{mM})$ exposure with or without thymol (20 $\left.\mu \mathrm{M}\right)$ up to $72 \mathrm{~h}$. The black arrow in (E-F) indicates that the seedlings of $\mathrm{NH}_{4} \mathrm{Cl}+$ Thymol treatment begin to grow significantly longer that $\mathrm{NH}_{4} \mathrm{Cl}$ treatment. Different lowercase letters indicate that the mean values of ten replicates are significantly different among different treatments $(P<0.05, \mathrm{ANOVA}$, LSD)

further evaluated the effect of thymol on oxidative injury in rice seedlings under ammonium stress. TBARS, a product of lipid peroxidation, indicates the degree of cell oxidative damage (Tsikas 2017). $\mathrm{NH}_{4} \mathrm{Cl}$ treatment significantly increased TBARS content in both root and shoot, an effect that was repressed by thymol (Fig. 2A and B). Thymol also remarkably ameliorated $\mathrm{NH}_{4} \mathrm{Cl}$-induced lipid peroxidation and loss of membrane integrity in leaves (Fig. $1 C$ and D), coinciding with the change of TBRAS content. Dead cell was labeled specifically with PI to emit red fluorescence. Ammonium stress led to strong PI fluorescence in root, especially in root tip that is responsible for root elongation (Fig. 2E). Adding thymol weakened PI fluorescence in $\mathrm{NH}_{4} \mathrm{Cl}$-treated root tip (Fig. $2 \mathrm{E})$, suggesting that thymol recovered cell viability to facilitate root growth under $\mathrm{NH}_{4} \mathrm{Cl}$ stress. Cell death in leaves was evaluated with Trypan blue in order to avoid the interference of chlorophyll autofluorescence signal. Thymol attenuated cell death in leaves under ammonium stress as well (Fig. 2F). These results suggested that thymol was able to attenuate $\mathrm{NH}_{4} \mathrm{Cl}$-induced phytotoxicity by weakening oxidative injury and cell death in rice seedlings.
Thymol suppressed PAO-dependent ROS production in rice seedlings upon ammonium stress

Endogenous ROS at low level acts as indispensable signaling to regulate plant development under normal growth conditions. However, environmental stimuli frequently trigger ROS accumulation that attacks lipids or proteins to cause oxidative injury followed by cell death (Gill and Tuteja 2010). Detection of tissue ROS in situ without damaging plant tissue not only helps to understand the relationship between ROS distribution and plant development but also avoids inevitable loss of intube assay of ROS content. We tracked total ROS in situ with specific probe DCFH-DA emitting green fluorescence. The control root showed weak fluorescent signals (Fig. 3A). Ammonium stress induced significant increase in total ROS signal in root, which could be remarkably decreased by thymol (Fig. 3A and B). Then we detected the level of $\mathrm{H}_{2} \mathrm{O}_{2}$ and superoxide radical $\left(\mathrm{O}_{2}{ }^{--}\right)$, two kinds of typical ROS, in rice seedlings. Thymol also decreased the level of $\mathrm{H}_{2} \mathrm{O}_{2}$ and $\mathrm{O}_{2}{ }^{-{ }^{-}}$in roots (Fig. 3C-F) and leaves (Fig. 3G-H) upon ammonium stress. Notably, ROS accumulation in the elongation zone of root tip was sensitive to ammonium or thymol (Fig. 3A, C, E). Therefore, thymol-attenuated oxidative 


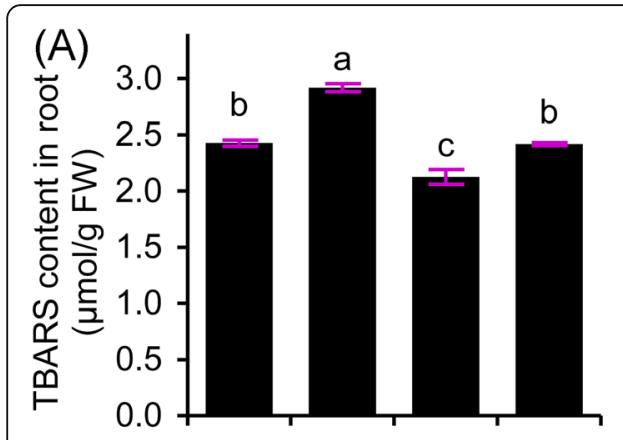

(B)

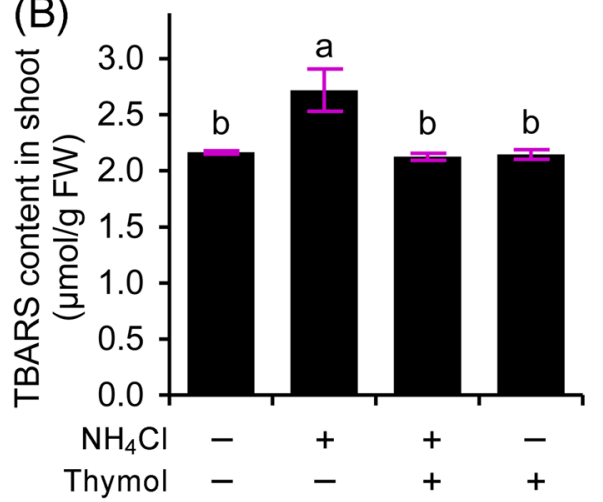

(C)

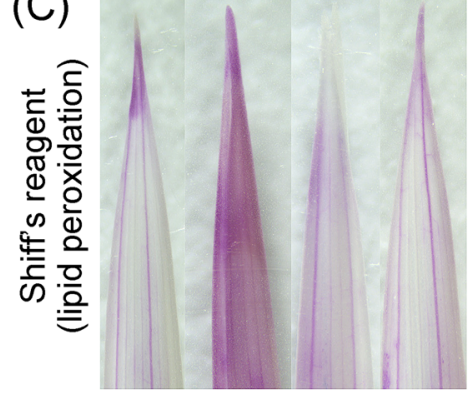

(D)

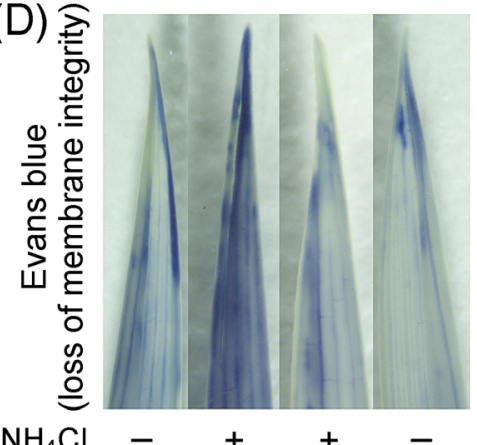

(E)

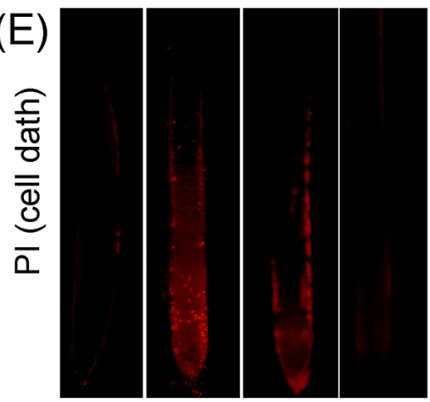

(F)

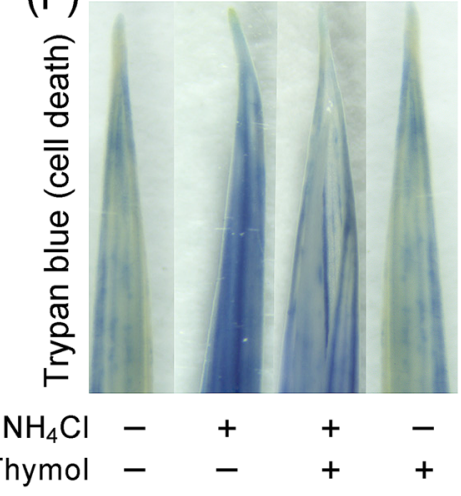

Fig. 2 Thymol attenuated ammonium-induced oxidative injury in rice seedlings. (A-B) Changes in TBARS content in root and shoot after $\mathrm{NH}_{4} \mathrm{Cl}$ exposure for $72 \mathrm{~h}$. (C-F) Detection lipid peroxidation in leaf (C), loss of membrane integrity in leaf (D), and cell death in root (E), and cell death in leaf (F) after $\mathrm{NH}_{4} \mathrm{Cl}(20 \mathrm{mM})$ exposure with or without thymol $(20 \mu \mathrm{M})$ for $72 \mathrm{~h}$. The black arrow in $(\mathrm{H})$ indicates the direction of root tip. Different lowercase letters in $\mathrm{A}-\mathrm{H}$ indicate that the mean values of ten replicates are significantly different among different treatments $(P<0.05$, ANOVA, LSD)

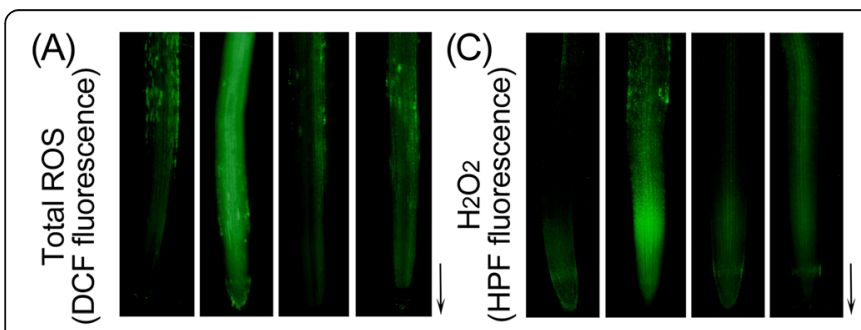

(B)

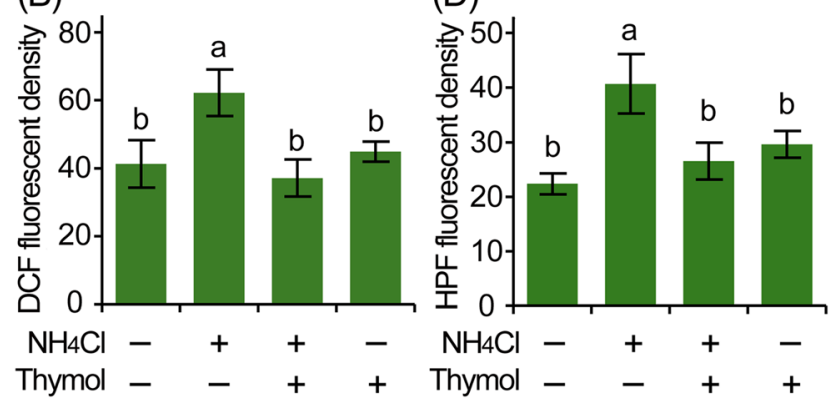

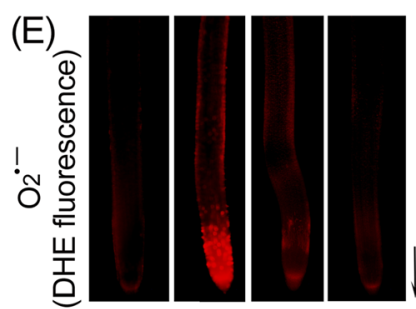

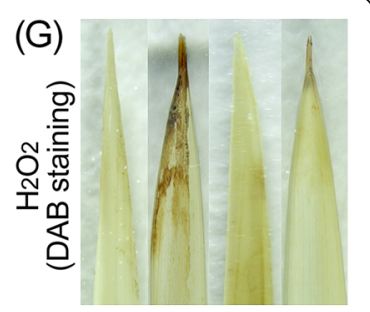

(F)

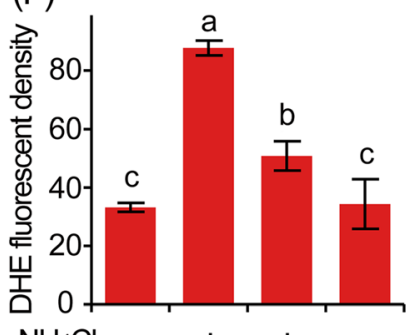

(H)
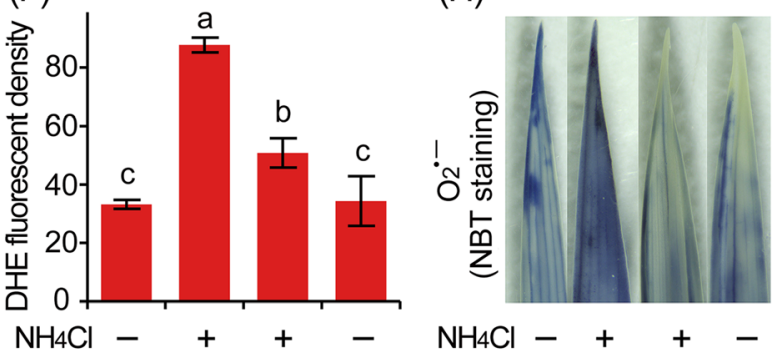

Thymol - -++ Thymol $-\quad++$

Fig. 3 Thymol repressed ammonium-induced ROS accumulation in rice seedlings. (A-B) Total ROS level in root. (C-D) $\mathrm{H}_{2} \mathrm{O}_{2}$ level in root. (E-F) $\mathrm{O}_{2}{ }^{-}$ level in root. (G) $\mathrm{H}_{2} \mathrm{O}_{2}$ level in leaves. ( $\left.\mathrm{H}\right) \mathrm{O}_{2}^{-}$level in leaves. The black arrows in $\mathrm{A}, \mathrm{C}$, and $\mathrm{E}$ indicates the direction of root tip. Different lowercase letters in $B, D$, and $F$ indicate that the mean values of three replicates are significantly different among treatments $(P<0.05$, ANOVA, LSD) 


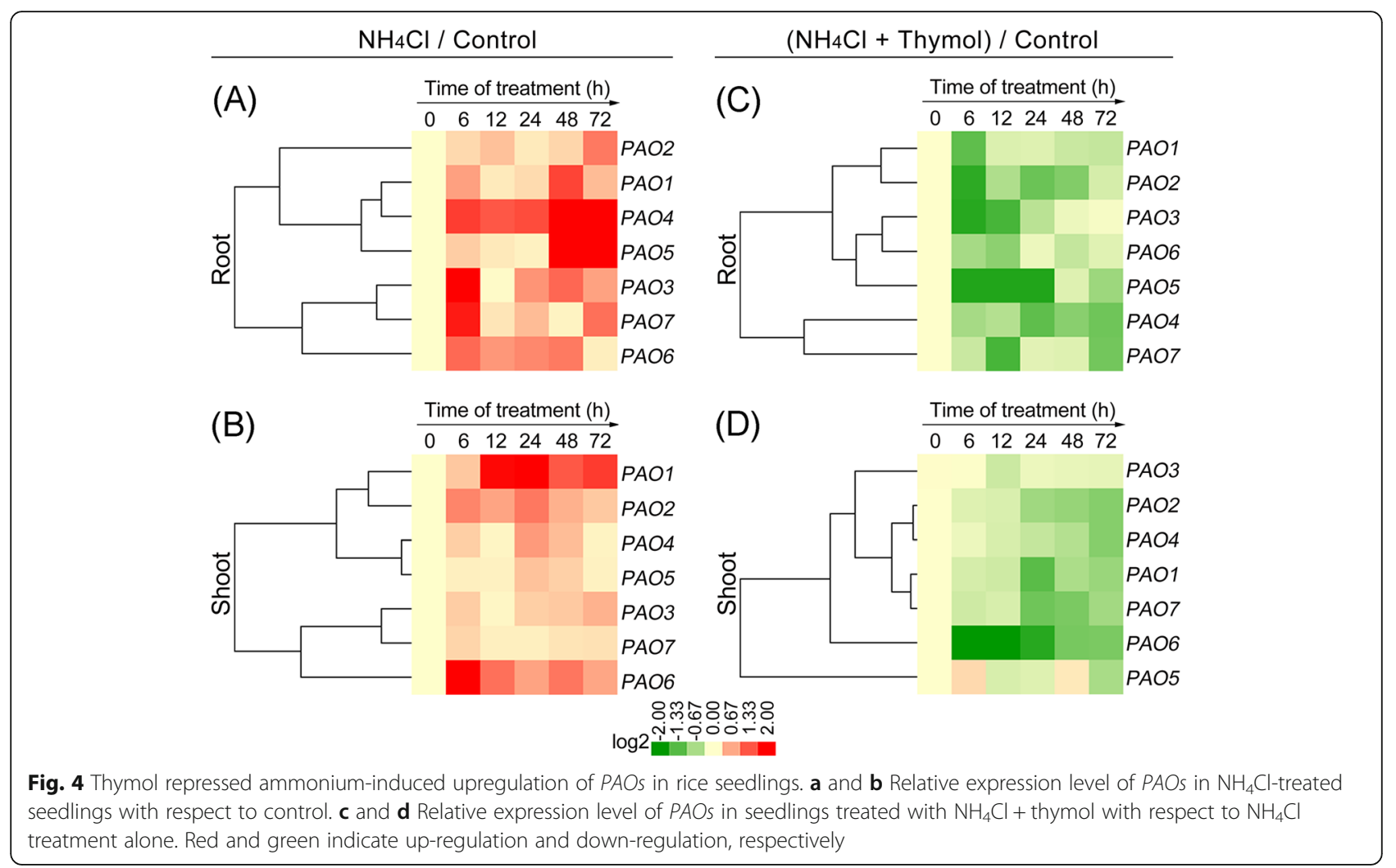

injury and cell death may resulted from the decrease in ROS level, further leading to the recovery of root growth upon ammonium stress. In mammalian immune responses, thymol-repressed ROS accumulation results from the scavenging of already accumulated ROS. First, thymol itself has the ability to quench ROS directly (Javan and Javan 2014). Secondly, thymol can active anti-oxidative system to help eliminate over-generated ROS in both mammals and plants (Em et al. 2015; Cheng et al. 2020). However, little is known about whether and how thymol prevents ROS overgeneration in cells under stress conditions. To address this question, we examined the effect of thymol on the expression of a set of PAOs that can produce ROS.

A total of seven $P A O$ genes have been identified from rice genome (Chen et al. 2016). Gene expression analysis suggested that ammonium treatment extensively upregulated the expression of $\mathrm{PAO1-7}$ in both root and shoot. And ammonium induced much higher expression level of PAOs in root than in shoot (Fig. 4a and b). In ammonium-treated root, the expression of PAO3 and PAO7 were clustered together, which seemed to be induced at early stages. PAO1, 2, 4, and 5 in another group were upregulated at later stages (Fig. 4a). Therefore, ammonium-induced ROS over-generation may partially attributed to the increase in the expression of PAOs. In both root and shoot, adding thymol downregulated the expression of PAOs compared with ammonium treatment alone (Fig. 4c and d). These results suggested that thymol prohibited ammoniuminduced upregulation of $P A O s$, further leading to the inhibition of ROS generation in rice seedlings.

\section{Thymol differentially modulated the expression of genes related to ammonium transport in rice seedlings}

Ammonium exposure remarkably induced the expression of two ammonium transporters, AMT1;1 and $A M T 1 ; 2$ in root (Fig. 5a). Meanwhile, the expression of CIPK23 and CBL1, two suppressors of AMTs, decreased upon ammonium exposure (Fig. 5a). However, adding thymol reversed the expression level of these genes in ammonium-treated root (Fig. 5a). And these genes showed similar expression patterns in shoot as compared to root (Fig. 5b). The relative expression abundance of these genes was relatively lower in shoot than in root, which may due to the direct exposure of root to treatment solution. Thus it can be suspected that thymol may modulate ammonium 


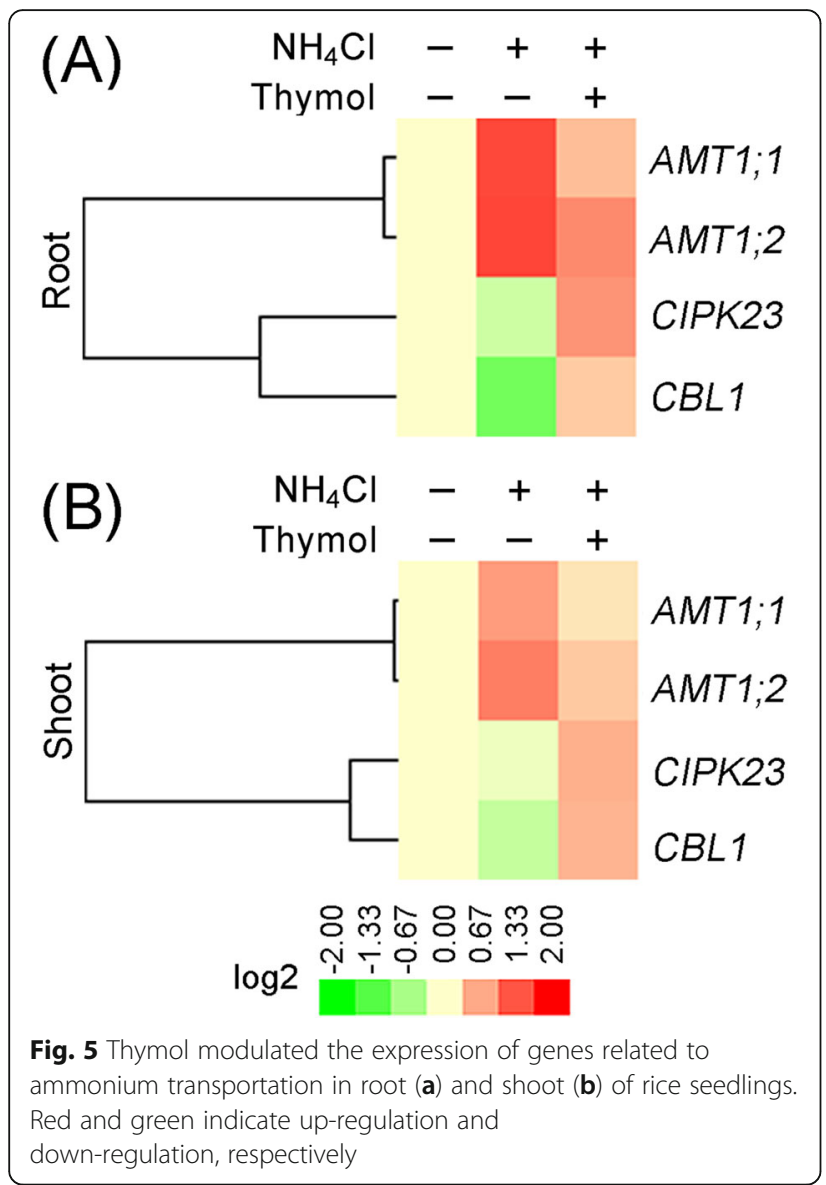

transportation to avoid ammonium overload in rice seedlings, leading to the attenuation of phytotoxicity.

To further conclude the regulatory mode of thymol, we performed Pearson correlation analysis among different parameters in root under ammonium or thymol treatment (Fig. 6). Root length was negatively correlated to TBARS, PI and ROS, respectively. ROS was positively correlated to TBARS and PI. The expression levels of PAOs were positively correlated to ROS, but negatively correlated to root length. Therefore, the decrease in PAOs-derived ROS may partially contribute to thymolattenuated oxidative injury, further resulting in the recovery of root growth under ammonium stress. The expression levels of $A M T 1 ; 1$ and $A M T 1 ; 2$ were positively correlated to PAOs expression, TBARS, PI, and ROS, respectively, but negatively correlated to root length. Therefore, ammonium overload mediated by AMT1;1 and $A M T 1 ; 2$ probably resulted in phytotoxicity, which could be attenuated by thymol.

Thymol stimulated the expression of both CIPK23 and $C B L 1$ in ammonium-treated roots, but CBL1 showed relatively stronger correlation with other parameters than CIPK23. It has been reported that CBL1 interacts with CIPK23 to suppress AMTs (Straub et al. 2017). Therefore, CBL1 may be a major target of thymol for the suppression of AMT1;1 and AMT1;2. The function of plant CBL is dependent on $\mathrm{Ca}^{2+}$ binding (Batistič and Kudla 2009). Thymol can facilitate $\mathrm{Ca}^{2+}$ influx into mammalian cells by directly activating $\mathrm{Ca}^{2+}$ channel (Lee et al. 2008). Therefore, thymol may regulate $\mathrm{CBL}$ through $\mathrm{Ca}^{2+}$-dependent pathway, which needs further studies. Thymolrepressed ammonium transportation may contribute to the decrease in PAOs expression and ROS generation, but we cannot exclude the possibility that thymol may directly regulate the expression of PAOs under ammonium stress. Our previous study has documented that stress-related cis-elements in plant $P A O$ promoters play a role in regulating $P A O$ expression under stress conditions (Wang et al. 2019). Thymolmediated physiological adaption involves the modulation of several stress-related signals (e.g. nitric oxide and $\mathrm{Ca}^{2+}$ ) (Lee et al. 2008; Wang et al. 2017). Therefore, it would be helpful to understand thymolregulated $P A O$ expression by establishing the possible link between thymol-modulate intercellular signal and stress-responsive elements in rice $P A O$ promoters.

\section{Conclusions}

In sum, we found that natural compound thymol had great potential to confer rice tolerance against ammonium stress. Compared with the capacity of thymol in triggering anti-oxidative system in mammalian cells, here we found a different role of thymol in decreasing ROS accumulation in plants. Thymol repressed $P A O$-derived $\mathrm{H}_{2} \mathrm{O}_{2}$ generation, leading to the alleviation of oxidative injury and cell death under ammonium stress. In addition, thymol was able to modulate the expression of ammonium transport regulatory genes, which might help rice plant avoid ammonium overload under excessive ammonium conditions. The detailed mechanism needs to be elucidated further, but our current results indicate that thymol has ability to regulate rice physiology to adapt to high ammonium environment. This may be applicable to modulate crop nutrient acquisition during rice production process. Rice seedlings grow frequently in anoxic conditions, and they are more sensitive to ammonium stress than adult plants. Thymol-induced ammonium tolerance may help rice survive in seedling stage under excessive ammonium environment. Thymol is natural essential oil that can be easily degraded in natural environment. We need to concern the possibility of the long-term usage of thymol in later stages of rice 


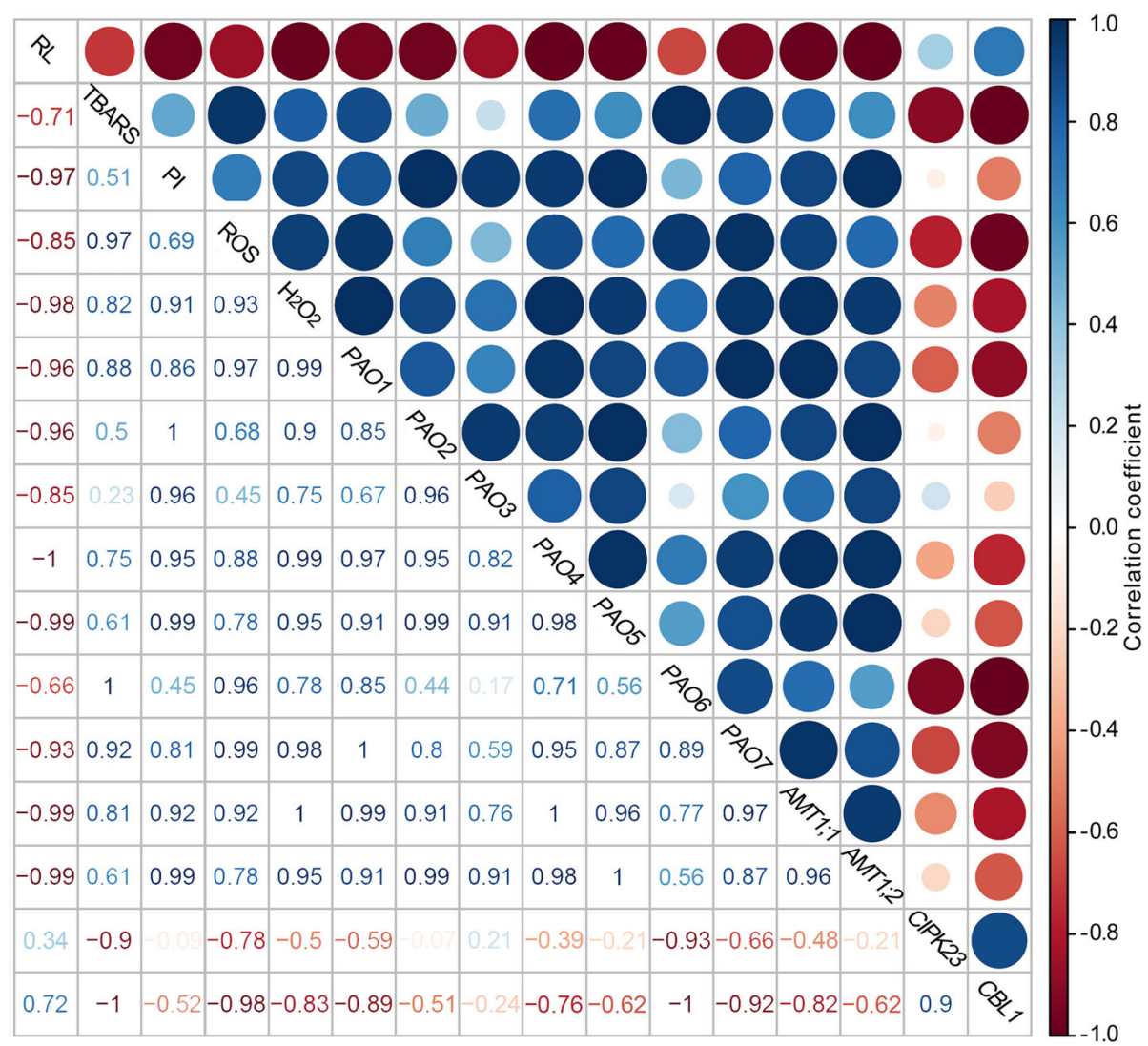

Fig. 6 Pearson correlation analysis of different physiological parameters in rice root under treatment of control, $\mathrm{NH}_{4} \mathrm{Cl}$, and $\mathrm{NH}_{4} \mathrm{Cl}+\mathrm{Thymol}$

growth. It has been demonstrated that microencapsulation or nanoencapsulation can improve the longterm effectiveness of essential oil (Shah et al. 2012; Zhang et al. 2020). Therefore, developing microencapsular or nanoencapsular thymol may have great potential to improve the efficiency of thymol in agriculture.

\section{Supplementary Information}

The online version contains supplementary material available at https://doi. org/10.1186/s43014-021-00053-1.

Additional file 1: Table S1. Sequences of primers used for qRT-PCR analysis of rice genes. Fig. S1. Dose-dependent effect of thymol on rice growth under ammonium stress conditions.

\section{Abbreviations}

$\mathrm{NH}_{4}^{+}$: Ammonium; ROS: Reactive oxygen species; PAO: Polyamine oxidase; $\mathrm{H}_{2} \mathrm{O}_{2}$ : Hydrogen peroxide; AMT: Ammonium transporter; CIPK23: Calcineurin B-like interacting protein kinase 23; CBL1: Calcineurin B-like binding protein $1 ; \mathrm{O}_{2}^{-}$: Superoxide radical; DCFH-DA: $2^{\prime}, 7^{\prime}$-dichlorofluorescein diacetate; DHE: Dihydroethidium; HPF: 3'-(p-hydroxyphenyl) fluorescein; PI: Propidium iodide; NBT: Nitro-blue tetrazolium; DAB: 3,3-diaminobenzidine;

TBARS: Thiobarbituric acid reactive substances; TBA: 1,3-diethyl-2thiobarbituric acid

\section{Acknowledgements}

We thanks Mr. Cunfa Xu from Central Laboratory in Jiangsu Academy of Agricultural Sciences for his help of stereoscopic microscopy study.

\section{Authors' contributions}

Jian Chen and Kai Guo designed the experiments and wrote the paper. Kai Guo, Guangchi An, and Ning Wang performed phenotypic and histochemical analysis. Bingdong Pang and Zhiqi Shi finished gene expression analysis. Jian Chen, Kai Guo, Hongwu Bai, and Li Zhang analyzed the data. Jian Chen, Kai Guo, Guangchi An, and Weimin Xu prepared the draft. The author(s) read and approved the final manuscript.

\section{Funding}

This work was supported by National Natural Science Foundation of China (31771705), The Central Government Guides Local Science and Technology Development Fund Projects (YDZX20203700003919), China Postdoctoral Science Foundation (2015 M570627), Program for Youth Innovation and Technology Team in University of Shandong Province (2019KJF016), Rizhao (vegetable) Science and Technology Innovation Center (Rikezi [2020] No.13), Special Science and Technology Project of Rizhao (2019CXZX2205), Shandong Academy of Science Fund for Distinguished Young Scholars (LKYZ2018-60), Science and Technology Project of Yantai (2019ZDCX016), Open Project Fund of the State Key Laboratory of Plant Cell and Chromosome Engineering (PCCE-KF-2019-05).

Availability of data and materials Not applicable.

Ethics approval and consent to participate Not applicable.

Consent for publication Not applicable.

Competing interests

The authors declare no conflict of interest. 


\section{Author details}

National Tobacco Cultivation and Physiology and Biochemistry Research Center / Key Laboratory for Tobacco Cultivation of Tobacco Industry, Henan Agricultural University, Zhengzhou 450002, China. ${ }^{2}$ Biology Institute, Qilu University of Technology (Shandong Academy of Sciences), Shandong Province Key Laboratory for Biosensors, Jinan 250014, China. ${ }^{3}$ Zaozhuang Technology Promotion Center of Agriculture and Agricultural Machinery, Zaozhuang 277800, China. ${ }^{4}$ Central Laboratory, Jiangsu Academy of Agricultural Sciences, Nanjing 210014, China. ${ }^{5}$ Institute of Food Safety and Nutrition, Jiangsu Academy of Agricultural Sciences / Laboratory for Food Quality and Safety-State Key Laboratory Cultivation Base of Ministry of Science and Technology, Nanjing 210014, China. ${ }^{6}$ College of Plant Protection, Shandong Agricultural University, Taian 271018, China. ${ }^{7}$ Supervision \& Testing Center for Processed Agri-products (Nanjing), Ministry of Agriculture, Nanjing 210014, China.

\section{Received: 22 September 2020 Accepted: 26 January 2021}

Published online: 05 March 2021

\section{References}

Alcázar, R., \& Tiburcio, A. F. (2014). Plant polyamines in stress and development: An emerging area of research in plant sciences. Frontiers in Plant Science, 5, 319.

Altman, A., \& Levin, N. (1993). Interactions of polyamines and nitrogen nutrition in plants. Physiologia Plantarum, 89(3), 653-658.

Basch, E., Ulbricht, C., Hammerness, P., Bevins, A., \& Sollars, D. (2004). Thyme (Thymus vulgaris L.), thymol. Journal of Herbal Pharmacotherapy, 4, 49-67.

Batistič, O., \& Kudla, J. (2009). Plant calcineurin B-like proteins and their interacting protein kinases. Biochimica et Biophysica Acta (BBA) - Molecular Cell Research, 1793(6), 985-992.

Chen, B., Li, W., Gao, Y., Chen, Z., Zhang, W., Liu, Q., ... Liu, J. (2016). Involvement of polyamine oxidase-produced hydrogen peroxide during coleorhiza-limited germination of rice seeds. Frontiers in Plant Science, 7, 1219.

Chen, G., Guo, S., Kronzucker, H. J., \& Shi, W. (2013). Nitrogen use efficiency (NUE) in rice links to $\mathrm{NH}_{4}{ }^{+}$toxicity and futile $\mathrm{NH}_{4}{ }^{+}$cycling in roots. Plant and Soil, $369,351-363$

Cheng, Y.-W., Kong, X.-W., Wang, N., Wang, T.-T., Chen, J., \& Shi, Z. (2020). Thymol confers tolerance to salt stress by activating anti-oxidative defense and modulating $\mathrm{Na}^{+}$homeostasis in rice root. Ecotoxicology and Environmental Safety, 188, 109894.

Em, E.-S., Ar, A.-A., Am, M., \& Aa, E. L. A. (2015). Thymol and carvacrol prevent cisplatin-induced nephrotoxicity by abrogation of oxidative stress, inflammation, and apoptosis in rats. Journal of Biochemical and Molecular Toxicology, 29(4), 165-172.

Esteban, R., Ariz, l., Cruz, C., \& Moran, J. F. (2016). Review: Mechanisms of ammonium toxicity and the quest for tolerance. Plant Science, 248, 92-101.

Gerendás, J., Zhu, Z., Bendixen, R., Ratcliffe, R. G., \& Sattelmacher, B. (1997). Physiological and biochemical processes related to ammonium toxicity in higher plants. Zeitschrift für Pflanzenernährung und Bodenkunde, 160(2), 239251.

Gill, S. S., \& Tuteja, N. (2010). Reactive oxygen species and antioxidant machinery in abiotic stress tolerance in crop plants. Plant Physiology and Biochemistry, 48(12), 909-930.

Hu, B., Wang, W., Ou, S., Tang, J., Li, H., Che, R., ... Chu, C. (2015). Variation in NRT1.1B contributes to nitrate-use divergence between rice subspecies. Nature Genetics, 47(7), 834-838.

Javan, A. J., \& Javan, M. J. (2014). Electronic structure of some thymol derivatives correlated with the radical scavenging activity: Theoretical study. Food Chemistry, 165, 451-459.

Lee, S. P., Buber, M. T., Yang, Q., Cerne, R., Cortés, R. Y., Sprous, D. G., \& Bryant, R. W. (2008). Thymol and related alkyl phenols activate the hTRPA1 channel. British Journal of Pharmacology, 153(8), 1739-1749.

Li, S.-M., Li, B.-Z., \& Shi, W.-M. (2012). Expression patterns of nine ammonium transporters in rice in response to $\mathrm{N}$ status. Pedosphere, 22(6), 860-869.

Livak, K. J., \& Schmittgen, T. D. (2001). Analysis of relative gene expression data using real-time quantitative PCR and the $2^{-\Delta \Delta C T}$ method. Methods, 25(4), 402-408.

Marchese, A., Orhan, I. E., Daglia, M., Barbieri, R., Di Lorenzo, A., Nabavi, S. F., .. Nabavi, S. M. (2016). Antibacterial and antifungal activities of thymol: A brief review of the literature. Food Chemistry, 210, 402-414.
Nagoor Meeran, M. F., Jagadeesh, G. S., \& Selvaraj, P. (2016). Thymol, a dietary monoterpene phenol abrogates mitochondrial dysfunction in $\beta$-adrenergic agonist induced myocardial infarcted rats by inhibiting oxidative stress. Chemico-Biological Interactions, 244, 159-168.

Shah, B., Davidson, P. M., \& Zhong, Q. (2012). Nanocapsular dispersion of thymol for enhanced dispersibility and increased antimicrobial effectiveness against Escherichia coli 0157:H7 and Listeria monocytogenes in model food systems. Applied and Environmental Microbiology, 78(23), 8448-8453.

Shi, H., Ye, T., \& Chan, Z. (2014). Nitric oxide-activated hydrogen sulfide is essential for cadmium stress response in bermudagrass (Cynodon dactylon (L). Pers.). Plant Physiology and Biochemistry, 74, 99-107.

Sonoda, Y., Ikeda, A., Saiki, S., Wirén, N. v., Yamaya, T., \& Yamaguchi, J. (2003). Distinct expression and function of three ammonium transporter genes (OsAMT1;7-1;3) in rice. Plant and Cell Physiology, 44(7), 726-734.

Straub, T., Ludewig, U., \& Neuhäuser, B. (2017). The kinase CIPK23 inhibits ammonium transport in Arabidopsis thaliana. Plant Cell, 29(2), 409-422.

Tsikas, D. (2017). Assessment of lipid peroxidation by measuring malondialdehyde (MDA) and relatives in biological samples: Analytical and biological challenges. Analytical Biochemistry, 524, 13-30.

USEPA/RIS (2014). Integrated risk information system. http://www.epa.gov/iris/. Accessed 7 Nov 2014.

Wang, T. T., Shi, Z. Q., Hu, L. B., Xu, X. F., Han, F. X., Zhou, L. G., \& Chen, J. (2017), Thymol ameliorates cadmium-induced phytotoxicity in the root of rice (Oryza sativa) seedling by decreasing endogenous nitric oxide generation. Journal of Agricultural and Food Chemistry, 65(34), 7396-7405.

Wang, Y., Ye, X., Yang, K., Shi, Z., Wang, N., Yang, L., \& Chen, J. (2019). Characterization, expression, and functional analysis of polyamine oxidases and their role in selenium-induced hydrogen peroxide production in Brassica rapa. Journal of the Science of Food and Agriculture, 99, 4082-4093.

Wei, T., \& Simko, V. (2017). R package "corrplot": Visualization of a correlation matrix (version 0.84). https://github.com/taiyun/corrplot.

Xie, Y., Mao, Y., Xu, S., Zhou, H., Duan, X., Cui, W., ... Xu, G. (2015). Heme-heme oxygenase 1 system is involved in ammonium tolerance by regulating antioxidant defence in Oryza sativa. Plant, Cell \& Environment, 38(1), 129-143.

Yang, Y., Fan, F., Zhuo, R., Ma, F., Gong, Y., Wan, X., ... Zhang, X. (2012). Expression of the laccase gene from a white rot fungus in Pichia pastoris can enhance the resistance of this yeast to $\mathrm{H}_{2} \mathrm{O}_{2}$-mediated oxidative stress by stimulating the glutathione-based antioxidative system. Applied and Environmental Microbiology, 78(16), 5845-5854.

Ye, X., Ling, T., Xue, Y., Xu, C., Zhou, W., Hu, L., .. Shi, Z. (2016). Thymol mitigates cadmium stress by regulating glutathione levels and reactive oxygen species homeostasis in tobacco seedlings. Molecules, 21(10), 1339.

Ye, X.-F., Xue, Y., Ling, T., Wang, Y., Yu, X.-N., Cheng, C.., ... Chen, J. (2017). Cinnamaldehyde ameliorates cadmium-inhibited root elongation in tobacco seedlings via decreasing endogenous hydrogen sulfide production. Molecules, 22(1), 15.

Zhang, T., Luo, Y., Wang, M., Chen, F., Liu, J., Meng, K., \& Zhao, H. (2020). Doublelayered microcapsules significantly improve the long-term effectiveness of essential oil. Polymers (Basel), 12(8), 1651.

\section{Publisher's Note}

Springer Nature remains neutral with regard to jurisdictional claims in published maps and institutional affiliations.

Ready to submit your research? Choose BMC and benefit from:

- fast, convenient online submission

- thorough peer review by experienced researchers in your field

- rapid publication on acceptance

- support for research data, including large and complex data types

- gold Open Access which fosters wider collaboration and increased citations

- maximum visibility for your research: over $100 \mathrm{M}$ website views per year

At $\mathrm{BMC}$, research is always in progress.

Learn more biomedcentral.com/submissions 